\title{
Educación experiencial en Psicología Clínica
}

Elizabeth Jorge

eli21jorge@gmail.com

\section{Macarena Guzmán}

macarenaguzman07@gmail.com

\section{Cristina González}

mcristinagonzalez@gmail.com

\section{Gladis Gentes}

gladisbeatriz.gentes@gmail.com

Docentes investigadores de la

Facultad de Psicología. Universidad Nacional de Córdoba, Argentina.
Integración de la docencia y la extensión /

Intervenciones

RECEPCIÓN: 24/06/16

ACEPTACIÓN FINAL: 10/10/16

\section{Resumen}

El presente trabajo busca reflexionar sobre la práctica de extensión como un espacio adecuado de educación experiencial para los alumnos de la cátedra de Psicología Clínica en la Facultad de Psicología de la Universidad Nacional de Córdoba. En primer lugar se describe en qué consiste el proyecto extensionista llevado a cabo hasta el momento. A continuación se exponen los cambios que implicó la decisión de incluir alumnos de grado en la práctica extensionista y los fundamentos teóricos que permitieron definir el tipo de formación que se busca para los estudiantes. Por último, se plantean breves comentarios sobre el análisis de la propia práctica docente.

\section{Resumo}

Este trabalho procura refletir sobre a prática da extensão como espaço adequado de educação experiencial para os alunos da disciplina de Psicologia Clínica na Faculdade de Psicologia da Universidade Nacional de Córdoba. Em primeiro lugar, descreve-se qual o fundamento do projeto de extensão realizado até agora. A seguir se expõem as mudanças que envolveram a decisão de incluir alunos de graduação na prática de extensão e os fundamentos teóricos que permitiram definir o tipo de formação que se aspira para os alunos. Finalmente, apresentamse breves comentários sobre a análise da própria prática docente.

\section{Palavras-chave}

- Prática

- Extensão

- Educação experiencial

- Orientação tutorial

- Educación experiencial

- Orientación de tutores 


\author{
el acompañamiento en los \\ procesos de aprendizaje de los \\ niños/as de escuelas primarias \\ generó vínculos que redundaron \\ en el apoyo integral del niño
}

\section{Introducción}

La sociedad le exige a las universidades, en relación con la formación que brindan a los estudiantes, una capacitación que permita un adecuado ejercicio de la profesión (González y Silva, 2008; Di Doménico, 2015). Es decir, se busca no solo que el título otorgado por las unidades académicas brinde la garantía de que el joven profesional está totalmente capacitado sino también el respaldo de una formación general y de una disciplina de trabajo con las cuales pueda hacer frente a los desafíos de la profesión en el contexto actual que le toca desempeñarse. Es por ello que se promueve un proceso de reflexión acerca de lo que se enseña y produce (González y Silva, 2008; Di Doménico, 2015). Hay acuerdo en sostener el espacio de la extensión como aquel que permite el encuentro entre la universidad y la sociedad (Arocena, 2010; Corvalán de Mezzano, 2010; Pacheco, 2012; Menéndez y otros, 2013). Sin embargo, tal vez es la función menos claramente definida, más diversamente entendida y más vulnerable a los cambios de las situaciones políticas internas y externas de la universidad (Pacheco, 2012).

Se parte de la idea que:

"la extensión no solo es un proceso de formación integral a través del cual se corresuelven problemáticas sociales definidas, con el aporte del conocimiento producido por la investigación, la reflexión y la crítica, sino que constituye un aporte fundamental a la planificación de la enseñanza formal universitaria y para la orientación, tematización de la investigación científica. Es, por lo tanto, la función que sirve de guía política a la institución y que garantiza la pertinencia social del trabajo de la universidad". (Pacheco, 2012:22)
Además, se considera que la actividad docente universitaria parte del supuesto de que en ella están articuladas la docencia, la investigación y la extensión. La primera se alude a la enseñanza; la segunda, a la producción de conocimientos; y la tercera, a la relación de la universidad con la comunidad donde se encuentra inserta (López, 2012). En este artículo se busca reflexionar sobre los desafíos que implicó incluir alumnos de grado que están cursando $4^{\circ}$ año de la carrera de Licenciatura en Psicología en una práctica extensionista.

\section{Presentación del proyecto de carácter extensionista}

La incorporación de los alumnos cursantes de la asignatura Psicología Clínica se hizo en el marco del proyecto que desarrollan las autoras, denominado "Orientación y contención a alumnos tutores". Esta propuesta surge a partir de la demanda con dos escuelas preuniversitarias que venían realizando un trabajo extensionista ${ }^{1}$ con los alumnos de final de secundaria. Estos estudiantes, llamados "alumnos tutores", realizan apoyo escolar a niños/as que asisten a otras dos escuelas públicas de nivel primario con el objetivo de disminuir las dificultades escolares. El acompañamiento en los procesos de aprendizaje de los niños/as de estas escuelas primarias generó vínculos que redundaron en el apoyo integral del niño. El acompañamiento "uno a uno" le brindó confianza a los niños/as, favoreció su autoestima y alentó el agrado por el estudio. En los alumnos tutores abrió horizontes vocacionales. El compromiso interinstitucional motivó el aprendizaje recíproco, afianzó lazos de vecindad y creó nuevos espacios para compartir. ${ }^{2}$
1) El Proyecto "Punto de Encuentro" es sostenido, en la actualidad, por las licenciadas Celeste Pedroni, Ivana
Sánchez y Mariana Echavarría, quienes coordinan la propuesta desde los respectivos colegios preuniversitarios donde se desempeñan. En su origen también fue coordinadora la licenciada Sandra Gezmet.
2) Esta frase fue expresado en el marco de una entrevista con la Licenciada Celeste Pedroni. 
Sin embargo, más allá de los beneficios de esta tarea, dado el vínculo establecido, se generan ciertas emociones, malestares y sentimientos que es necesario contener. Esta circunstancia lleva a solicitar la intervención de la cátedra de Psicología Clínica. La consideración del pedido desde ambos colegios y el análisis diagnóstico permitieron definir una estrategia de intervención para la orientación y contención a alumnos tutores". El servicio que se ofrece consiste en: a) facilitar un espacio que permita expresar las fantasías, creencias, ideas y expectativas previas al desempeño del rol de tutor; b) identificar las características personales que facilitarían el desempeño del rol;

c) desarrollar estrategias que permitan la construcción del vínculo con el niño; d) acompañar la elaboración de ansiedades básicas que la práctica genera; e) recoger las expresiones de los tutores con relación a lo que le significó la experiencia; y f) brindar un espacio de contención de las emociones producidas por la separación y disolución del vínculo.

Esta práctica extensionista se realiza dentro de la mencionada cátedra desde el año 2009 y continúa sin interrupciones hasta la actualidad. El equipo interviniente se conformó con algunos miembros de la cátedra, específicamente con docentes, adscriptos y ayudantes alumnos, ${ }^{3}$ quienes son los que organizan los encuentros con los tutores. Dentro de éstos cabe mencionar un total de cuatro reuniones que son entendidas como "unidades funcionales". Es decir que cada una de ellas se desdobla en dos o más encuentros, dependiendo de las necesidades del grupo de alumnos tutores que se acompaña. Los ejes sobre los que se desarrollan las actividades pensadas para cada una de estas reuniones son cuatro, a saber: construcción del rol de tutor, diseño del primer día del apoyo escolar, seguimiento, y evaluación, y cierre con los alumnos tutores. Se describe brevemente cada eje: a) Construcción del rol de tutor: en estos primeros encuentros se trabaja sobre lo que es "ser tutor". La tutoría, al ser un proceso de acompañamiento que debería estar presente durante la formación de los estudiantes, se manifiesta mediante la atención personalizada a un alumno o a un grupo reducido de alumnos para mejorar el rendimiento académico, solucionar problemas escolares, desarrollar hábitos de estudio, trabajo, reflexión y convivencia social (Peinado-Guevara y otros, 2011). Esto posibilita que los alumnos tutores ayuden a otros a mejorar su calidad en el proceso de enseñanza- aprendizaje por medio de la adquisición y desarrollo de hábitos, valores y habilidades. Luego de definir en conjunto lo que se entiende por alumno tutor, abordando la idea que es un rol "a construir" con características propias de cada uno de los estudiantes, se describe el modo en que se ha trabajado hasta el momento.

b) Diseño del primer día del apoyo escolar: se prevén dos actividades principales. En la primera se indaga sobre las expectativas, las escenas temidas y las fantasías en el encuentro con el futuro niño tutoreado. En la segunda actividad se comienzan los diseños de cómo se llevará a cabo el encuentro efectivo con el niño, es decir, la planificación de estrategias lúdicas para favorecer el vínculo y la confianza.

c) Seguimiento: consiste en dos tipos principales de actividades. En una de ellas se plantean espacios de supervisión, donde se promueve la expresión de los estudiantes en torno a cómo se está desarrollando la práctica. Allí pueden manifestar logros, problemas suscitados, fantasías, temores, etc. En el otro tipo de actividades se busca, mediante diferentes técnicas lúdicas (armado de redes por medio de hilo de lana) y gráficas (dibujo libre y espontáneo), la reflexión sobre el desempeño del rol y la construcción del vínculo con el niño tutoreado.

d) Evaluación y cierre con los alumnos tutores: se consideran dos tipos de evaluaciones: individual y grupal. La primera se hace por escrito, donde se indaga sobre el espacio de contención y acompañamiento y la valoración de la experiencia. Entre las preguntas se destacan los aportes de los encuentros al rol del tutor, si deben continuar los espacios de contención y acompañamiento, el sentimiento de los adolescentes en el rol de tutor, y las estrategias que les resultaron eficaces en la experiencia. La segunda evaluación es grupal, y en la misma se lleva a cabo una técnica que permita rescatar aspectos de la experiencia realizada.

La modalidad con la que se trabajan estas actividades con los tutores es grupal, con distintas dinámicas tendientes a la expresión de los alumnos tutores. Se prevé una cantidad de 20 alumnos aproximadamente por encuentro. La duración estipulada para cada una de las reuniones es de dos horas.

Dentro de las etapas descriptas por Corvalán de Mezzano (2010) en el trabajo institucional, se puede decir que nos encontramos en la etapa de intervención propiamente dicha, cuyo objetivo general es intentar transformaciones desde y para el conjunto de miembros ( $p$. 45) y, en el caso de la práctica que aquí se describe, el conjunto de alumnos tutores. A partir de esta experiencia es que desde inicios
3) La cátedra se compone de doce docentes en total (profesor titular, profesores adjuntos y profesores asis- tentes), más colaboradores en calidad de adscriptos y ayudantes alumnos ad honorem. 


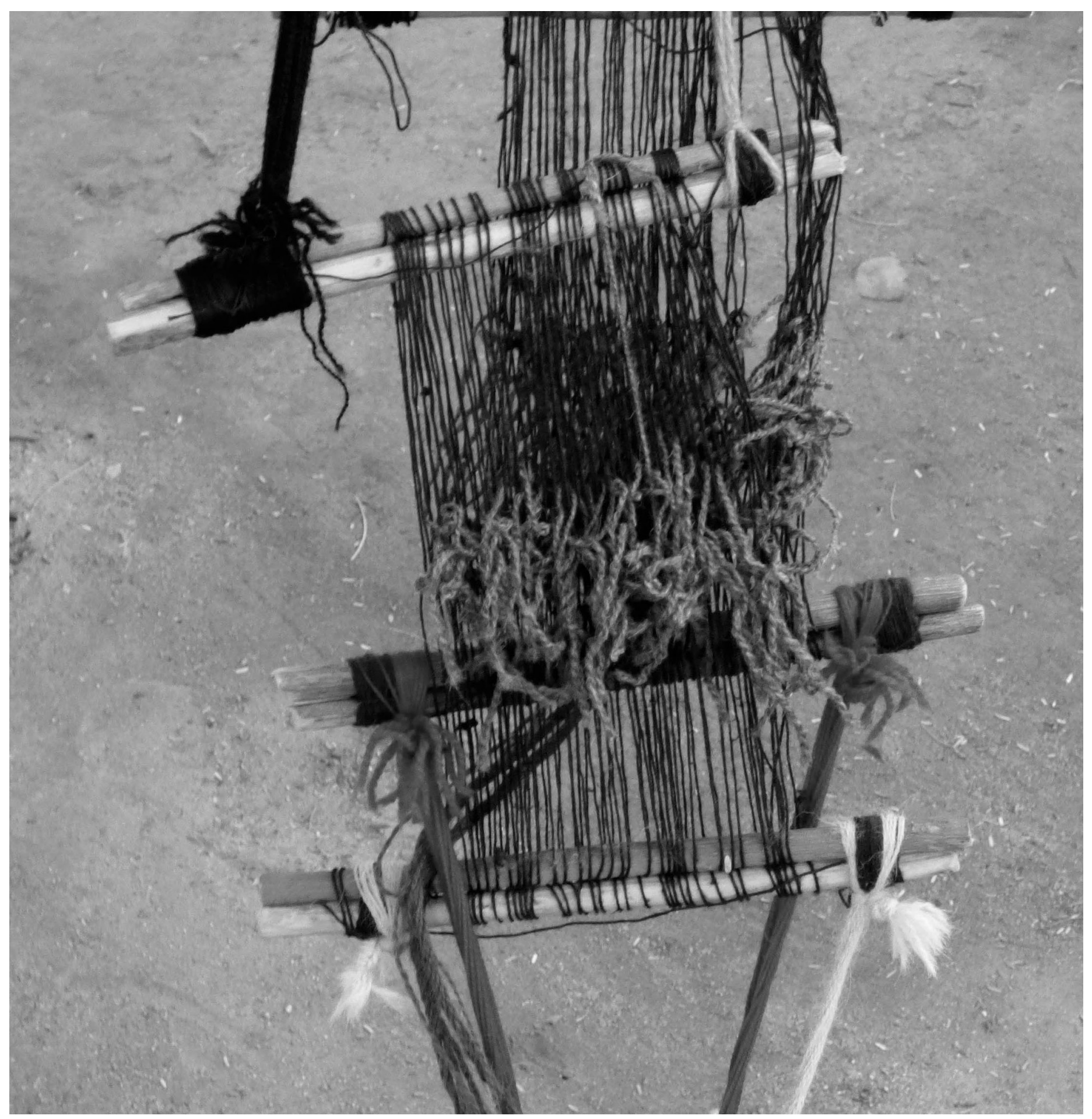

(C) Block Micaela 
del presente año se decide incluir a alumnos cursante de la asignatura, con la finalidad de promover la observación del trabajo clínico y la vivencia de articular teoría y práctica en el servicio a la comunidad.

Esto nos lleva a la reflexión sobre la formación académica y las posibilidades de intervención que posibilita. Siguiendo a Freire (1984), podemos entender la extensión universitaria no como mera transmisión de información y conocimientos académicos sino como un diálogo y un proceso creador, abierto a la pregunta y a la esperanza.

En este sentido, retomamos las preguntas planteadas por Torres y otros:

"¿la práctica de la extensión universitaria se constituye como práctica de formación?, ¿contribuye en la construcción de una identidad profesional comprometida con la realidad social? Ante las unánimes respuestas positivas que la experiencia reflexionada de cada uno ha generado: ¿sería posible pensar/ crear un espacio curricular para la integración de prácticas de extensión en los Planes de Estudio?". (2011:4)

La práctica extensionista que desarrollamos (al igual que muchas de las experiencias de este tipo) no está incluida en el currículo de la carrera, por lo que gran parte de los alumnos universitarios de nuestra Facultad transita una formación curricular obligatoria y solo algunos pocos añaden un voluntariado opcional. Es importante reflexionar en este punto acerca de que, en la práctica profesional, se nos plantean desafíos cotidianos a raíz de la complejidad para la cual no preparan los planes de estudios teóricos y disciplinarios. Es decir, si

"la complejidad de la realidad nos atraviesa, nos dificulta, nos limita, nos moviliza ¿en qué medida somos capaces de actuarla y de transformarla?, ¿cómo se relacionan estos impactos con un plan de estudios académico?" (Torres y otros, 2011:1).

\section{De la formación intraaulas a una formación en-contexto}

Hasta el momento, la práctica extensionista que se venía desarrollando se podría pensar como una de las formas de prestación de servicios universitarios posible para concretar la transferencia de conocimiento al medio social y, al mismo tiempo, para constituir una vía de retroalimentación de los conocimientos profesionales para renovar y actualizar la calidad docente (Corvalán de Mezzano, 2010).

La cátedra de Psicología Clínica, donde se desempeñan las autoras de este artículo, entiende la necesidad de formar a los alumnos cursantes en los cuatro ámbitos de intervención: la prevención, el diagnóstico, el tratamiento y la investigación en sus variados campos de aplicación y no solo en el tradicional campo hospitalario.
Esta visión de la actividad clínica se sostiene en la red tejida por el método clínico entendido como "el análisis del caso singular", sea ésta la singularidad de un sujeto, una pareja, un grupo, una familia, una institución, o una comunidad. El término singular no alude a individualidad sino a las particularidades exclusivas que se dan en esa situación. La maestría en el uso del método es lo que caracteriza la mirada y escucha clínica cualquiera sea la geografía donde recaiga. La comprensión del caso solamente puede ubicarse en la intersección de la práctica con la teoría. Se entiende que una práctica sin teoría queda vacía de fundamentos y una teoría sin corroboración con lo observable puede convertirse en dogma. La incorporación de los alumnos de la cátedra a la práctica extensionista les posibilitaría observar y reflexionar sobre el quehacer del psicólogo clínico en el marco de un proyecto que vincula la universidad con la comunidad. Dentro de las motivaciones expresadas por el grupo de estudiantes que se mostraron interesados en esta práctica, se encontraron:

a) Poder observar a los psicólogos "en acción", ya que participarían de los encuentros de supervisión con los alumnos tutores.

b) Al no tener que guiar los encuentros con los tutores, tendrían la oportunidad de ensayar posibilidades sobre cómo se podría intervenir. c) Observar el accionar del psicólogo clínico fuera del ámbito tradicionalmente asignado: "el consultorio".

d) Dado que están más cerca del egreso universitario, la práctica le ofrece un ejemplo del ejercicio profesional.

Siguiendo a Urrutia (2010), en la dinámica experiencial ofrecida a los alumnos de la cátedra se pueden identificar factores que definieron el método, entre ellos:

a) Se cambió el ambiente cotidiano de aprendizaje y se lo ubicó en un ambiente nuevo: sea en el colegio preuniversitario o la supervisión con las docentes.

b) Se buscaron canales nuevos para transmitir el problema/tema a abordar utilizando el recurso lúdico, gráfico y observacional para el abordaje del problema.

c) Se controlaron las variables como tiempo, recursos y nivel de dificultad, para generar en el alumno una necesidad de solucionarlo. Además, se buscó que el grupo forme parte de la dinámica del problema que acercan los alumnos tutores en la supervisión para acentuar la necesidad de generar conocimiento nuevo.

d) Se hizo una socialización abierta y reflexiva sobre los puntos emergentes de las supervisiones, de modo que cada alumno arribara a conclusiones sobre los tema/problema trabajados por medio de la ayuda del docente. Las conclusiones son individuales y el conocimiento obtenido también.

e) El proceso de aprendizaje estuvo dado por la voluntad del alumno a través del ritmo de asimilación y aprendizaje, lo cual está marcado por la problemática abordada. 


\section{4. ¿Por qué una práctica con carácter extensionista para los alumnos de grado?}

En el cursado de la materia de Psicología Clínica los alumnos pueden optar por prácticas en diversas instituciones o seminarios teórico-prácticos. Tal como se mencionó, durante este año se decidió abrir la convocatoria a los estudiantes de la materia para incorporarse como observadores en el proyecto extensionista que llevan a cabo las docentes autoras de este texto.

Esta convocatoria implicó para las docentes diseñar una innovación curricular, tal como lo entiende Susana Celman, es decir:

"hacerse cargo de focalizar, problematizar, nombrar y resignificar, desde otro lugar, las prácticas habituales y cotidianas. Es pensar nuevas relaciones en un territorio habitado, conocido y probablemente naturalizado. Es atreverse a imaginar lo no existente como intento de superación de lo actual, como propuesta de acción, como proyecto a construir en un espacio y un tiempo determinados, contando con ciertas condiciones que lo hacen posible y riesgoso". (2013:13)

Se diseñó una propuesta que incluyó una instancia previa de capacitación, donde se trabajaron ejes tales como: qué es la extensión universitaria, la presentación del proyecto, las técnicas a utilizarse en la práctica (observación, registro, reflexión, sistematización), la mirada clínica en el espacio educativo, entre otros. Luego de estos encuentros, los alumnos acompañaron a las docentes en la implementación del proyecto extensionista. De esta manera, para el equipo interviniente implicó la posibilidad de repensar lo transitado hasta el momento y plantearse preguntas orientadoras. Por una parte, para nosotras era clara la diferencia entre nuestra práctica enmarcada en el área clínica dentro de un espacio educativo y la del quehacer de un psicólogo educativo. ¿Cómo transmitir a los alumnos esta diferencia? Durante la capacitación se trabajaron textos seleccionados previamente por los docentes pero también se motivó a los estudiantes para que hicieran su propia búsqueda, de acuerdo con sus intereses, sobre este tema, de manera de poner luego en discusión esos aportes. Esto también permitió discernir el rol del psicólogo clínico en un proyecto extensionista, marco desde el cual se le imprimen características particulares. Por otra parte, se hizo foco en aquellas herramientas que va adquiriendo a lo largo de la formación (observación, registro, reflexión) para ejercitarlas durante la participación de los estudiantes.

Se buscó que la participación de los alumnos se convirtiera en una "educación experiencial", la cual se refiere a una clase particular de aprendizaje. Siguiendo a Camilloni, puede definirse como una estrategia de enseñanza que está destinada a relacionar el aprendizaje académico con la vida real. Para ello, se propone al alumno realizar actividades en las que pone a prueba las habilidades y los conocimientos teóricos que posee en una situación auténtica, de la vida real. Pero también se le pide que evalúe sus consecuencias, enriquezca esos conocimientos y habilidades, identifique nuevos problemas y fije prioridades en cuanto a las urgencias de su solución.

En la participación de los alumnos de este proyecto se habilitó el encuentro entre distintos actores sociales, lo cual devino en una experiencia educativa donde la extensión es acción intersubjetiva de problematización, concientización, reflexión y cambio (Rafaghelli, 2013). Solo de esta manera el estudiante aprende a usar los conocimientos adquiridos en el trabajo, dado que aprende en las condiciones sociales de la utilización auténtica de las nociones. Es decir, se trata de un verdadero aprendizaje experiencial, por el que se logran simultáneamente fines comunitarios y educativos (Camilloni, 2013).

En la supervisión de los estudiantes, luego de la experiencia de participación en el proyecto (asistieron a los espacios de contención y orientación a los alumnos tutores en calidad de observadores no participantes), los educandos pudieron contrastar sus ideas previas con lo acontecido en la realidad. En primer lugar, se encontraron con las dificultades para observar y registrar al mismo tiempo a un grupo de personas que por momentos se superponían en los decires. A partir de ello se plantearon posibles estrategias para seguir entrenando y superar los obstáculos encontrados. En segundo lugar, reflexionaron sobre la importancia de considerar sus propias emociones y el registro corporal como posibles intervinientes en su rol. A partir de estas elaboraciones se preguntaron sobre el marco teórico de referencia, no al modo de "encajar teoría y práctica", sino empezando a contemplar qué de la teoría respondía a lo observado. Asimismo, pudieron comprender de manera más global la implicancia del rol profesional en un proyecto extensionista, donde cada una de las intervenciones que se hacen es para favorecer el funcionamiento de una propuesta que nos conecta con las necesidades de un grupo de la sociedad. Se considera que con en este tipo de aprendizaje los alumnos pudieron poner en juego sus conocimientos, sus principios éticos y sus valores, sus destrezas y habilidades. Tal como lo sostiene Camilloni, los educandos pueden rever y poner en práctica aquellos conocimientos que corresponden a su carrera específica en lo que hace a su formación general, su conocimiento de teorías psicológicas, así como de leyes y normativas de aplicación específica en el campo de trabajo en el que se está actuando. Al mismo tiempo, el estudiante debe comprometerse con los valores y los principios éticos del plan de acción social en el que participa. $\mathrm{E}$, igualmente, se espera que desarrolle y demuestre el dominio de las destrezas generales y específicas que son necesarias para cumplir las tareas y lo que es indispensable.

Por último, se promovieron instancias donde se le solicitó que autoevalúen sus saberes, valores y destrezas para ponerlas con confianza al servicio de otros (Camilloni, 2013). 
Este aprendizaje se convirtió en una verdadera experiencia que, siguiendo a Skliar y Larrosa (2009), puede describirse como eso que le pasa al sujeto que aprende, es ese acontecimiento que le permite ser reflexivo, transformarse y repensarse para regresar a su actuar y mejorarlo según las determinaciones del contexto. Es un ir y venir sobre lo actuado, donde existe la posibilidad de ser como sujeto que aprende, abierto o cerrado, pero determinante en su actuación sobre su formación y transformación.

A partir de esta experiencia se produjeron nuevos saberes vinculados a las necesidades de actores e instituciones sociales. Tal como lo afirman Iglesias et al. (2015), no un conocimiento que solo sirva para llevar a los claustros o "pasear" por jornadas y congresos, sino un conocimiento que "se quede" y sea usado en el contexto que lo ha generado. La sistematización final que los estudiantes debieron realizar al concluir su práctica tuvo este objetivo como orientador. La reflexión y la articulación teórica implementadas por los alumnos de la cátedra contribuyeron no únicamente de modo individual a su formación sino que implicaron una oportunidad más de elaboración conjunta con el equipo docente que interviene desde la cátedra.

En este sentido, es importante reflexionar que, como docentes, formamos a futuros profesionales de la salud en el campo de la psicología. La formación vivencial o educación experiencial es poco integrada a los procesos y sistemas educativos actuales, quizás por la veneración y la tradición oral que en las aulas aún se conserva (Kruger, 2005, citado en Padierna Carmona y González Palacio, 2013). Sin embargo, la complejidad de las demandas por parte de la sociedad hoy exige a las universidades que, en tanto instituciones formadoras, capaciten a los psicólogos en nuestro caso para responder a demandas concretas de intervención. Es por ello que hacemos nuestras las palabras de Carrera:

"Pensar nuevos escenarios de práctica, problematizar la realidad de la que formamos parte, son pasos ineludibles para lograr una formación profesional centrada en la comunidad" (2015:36).

\section{Palabras finales}

La propuesta de incluir a alumnos de grado en la práctica extensionista que se viene desarrollando nos llevó a reflexionar y analizar la propia práctica docente desde los distintos dispositivos de formación que la Universidad propone. Esto condujo, por un lado, a una transformación del rol docente, tal como lo plantea Tommasino (2008), quien sostiene que se "reconfigura el rol docente cuando cambiamos el lugar-aula por la realidad misma" (Guzmán et al., 2015). Y por otro, implicó redefinir qué tipo de experiencia se buscaba ofrecer a los estudiantes de la asignatura de Psicología Clínica. En este punto, se hizo foco en brindar la oportunidad de involucrarse en lógicas institucionales diversas y en las necesidades de los actores sociales que forman parte del proyecto.
Es decir, se promueve una transformación por fuera del aula operando en el campo social del cual emergen los problemas y desafíos por los cuales es convocado el equipo de la cátedra de Psicología Clínica y para el que se intenta ofrecer a los alumnos una formación in situ.

De esta manera, y siguiendo las ideas expresadas por Castellanos García (2015), decimos que esta transformación de la práctica extensionista que se venía desarrollando busca comprender las necesidades de la sociedad del conocimiento a través del procesamiento de la información, de manera tal que tienda a lograr el aprendizaje máximo, estimular la creatividad y desarrollar la capacidad de iniciar el cambio y enfrentarse a él. Esto conduce a que, desde el rol docente, se reflexione sobre el quehacer cotidiano y se busquen métodos o herramientas que colaboren en este proceso. Por otra parte, permite aprender en forma colaborativa y desarrollar nuevas habilidades dentro de la disciplina (en este caso, la Psicología).

En consecuencia, la práctica extensionista tal como ha sido descripta en este artículo se constituye en una práctica integral, dado que implica un tipo particular de articulación entre los procesos de aprendizaje y enseñanza, investigación y extensión, y que involucra a distintos actores (adolescentes, adultos, docentes, alumnos). De este modo, la extensión es entendida como un proceso dialógico y crítico que redimensiona a la enseñanza, al aprendizaje y a la investigación (Tommasino, 2008). 


\title{
64
}

\author{
se hizo foco en brindar la oportunidad de \\ involucrarse en lógicas institucionales diversas \\ y en las necesidades de los actores sociales \\ que forman parte del proyecto
}

\section{Referencias bibliográficas}

Arocena, R. (2010). Curricularización de la extensión: ¿porqué, cuál, cómo? Cuadernos de Extensión N 1. Uruguay: Universidad de la República.

Camilloni, A. (2013). La inclusión de la educación experiencial en el currículo universitario. En Menéndez, G. y otros, Integración docencia y extensión. Otra forma de aprender y de enseñar. Santa Fe: Ediciones UNL.

Celman, S. (2013). Prólogo. En Menéndez G. y otros, Integración docencia y extensión. Otra forma de aprender y de enseñar. Santa Fe: Ediciones UNL.

Carrera, L. (2015). Importancia de las propuestas curriculares en la formación de los profesionales de la salud. Disponible en: https://bibliotecavirtual.unl.edu.ar/ojs/ index.php/Extension/article/viewFile/4587/6978

Castellanos García, M. A. E. (2015). Las comunidades de práctica, como una estrategia de práctica docente. Académicus, 1 (7), pp. 6-14

Corvalán de Mezzano, A. (2010). Psicólogos institucionales trabajando. La psicología institucional en docencia, investigación y extensión universitaria ( $2^{\circ}$ ed.). Buenos Aires: Eudeba.

Di Doménico, C. (2015). Formación de psicólogos, aristas de la calidad. Psiencia, Revista Latinoamericana de Ciencia Psicológica, 7 (1), 124-132.

Freire, P. (1984). ¿Extensión o comunicación? La concientización en el medio rural. Madrid: Siglo XXI Editores.

González, M. del L. y Silva, M.M. (2008). Formación universitaria basada en el desarrollo de competencias profesionales. Aproximación desde la carrera de Psicología de la Universidad Católica del Uruguay. Ciencias Psicológicas, II (2), 155-162.

Iglesias, I.; Denegri, A.; Espinel Maderna, M.C.; Scharagrodsky, C.J.; Roldan, L.Á y Fernández Knudsen, M. (2015). Participación de estudiantes de psicología y Trabajo social en extensión universitaria. Hacia la construcción del conocimiento Profesional en comunidades de práctica. Memorias VII Congreso Internacional de Investigación y Práctica Profesional en Psicología, XXII Jornadas de Investigación, X Encuentro de Investigadores en Psicología del MERCOSUR. Tomo1. Buenos Aires: Universidad de Buenos Aires.
Jorge, E.; Guzmán, M.; Gentes, G. y González, C. (2016). La práctica extensionista del docente universitario. Reconfiguración del rol en la construcción de la demanda. Revista EXT, (7). Disponible en: http://revistas.unc.edu.ar/index.php/ext/article/ view/14449/14534

López, M.L. (2012). Extensión universitaria: situación actual y aportes metodológicos. San Salvador de Jujuy: Universidad Nacional de Jujuy, Universitaria de Jujuy. Menéndez, G. y otros (2013). Integración docencia y extensión. Otra forma de aprender y de enseñar. Santa Fe: Ediciones UNL.

Pacheco, M. (2012). Reflexiones en torno a la construcción del espacio de la extensión universitaria hoy. Cuadernos de Educación, 21-30.

Padierna Carmona y González Palacio (2013). La metodología experiencial en la Educación Superior. Disponible en: http://revistas.usbbog.edu.co/index.php/ Itinerario_educativo/article/view/475/369

Peinado-Guevara, H. J. y otros (2011). Impacto de la tutoría escolar en la proyección de los estudiantes adultos en el nivel medio superior, modalidad semi-escolarizada. Revista de Sociedad, Cultura y Desarrollo Sustentable. Ra Ximhai, 7 (1). Rafaghelli, M. (2013). La dimensión pedagógica de la extensión. En Menéndez, G. y otros (2013). Integración docencia y extensión. Otra forma de aprender y de enseñar. Santa Fe: Ediciones UNL.

Tommasino, H. (2008). Generalización de las prácticas integrales. Los aportes de la Extensión para su implementación. Disponible en: psico.edu.uy/sites/default/ files/tommasino_practicas_integrales.pdf

Torres, F. M. y otros (2011). La extensión como experiencia de formación. Ponencia en el XI Congreso Iberoamericano de Extensión Universitaria. Santa Fe.

Skliar, C. y Larrosa, J. (Comp.). (2009). Experiencia y alteridad en educación. Rosario: Homo Sapiens Ediciones.

Urrutia, P. (2010). La educación experiencial como herramienta en el aula de clase. Vestigium. Revista Académica Universitaria, 4, 75-80. 